\title{
Use of continuous intrathecal baclofen in hereditary spastic paraplegia
}

This article was published in the following Dove Press journal:

Clinical Audit

15 December 2015

Number of times this article has been viewed

\author{
Jessie Khera' \\ Nadine E Andrew' \\ Dominique A Cadilhac ${ }^{1,2}$ \\ Tara Purvis' \\ Michael C Fahey 3,4 \\ Hyam Barry Rawicki' \\ 'Department of Medicine, Faculty \\ of Medicine, Nursing and Health \\ Sciences, Monash University, Clayton, \\ ${ }^{2}$ Stroke Division, Florey Institute \\ of Neurosciences and Mental \\ Health, Heidelberg, ${ }^{3}$ Department \\ of Paediatrics, Faculty of Medicine, \\ Nursing and Health Sciences, Monash \\ University, Clayton, ${ }^{4}$ Department \\ of Medicine, Melbourne University, \\ Parkville, VIC, Australia
}

Correspondence: Hyam Barry Rawicki Monash Children's Hospital, Monash Medical Centre, 246 Clayton Road, Clayton, VIC 3168, Australia $\mathrm{Tel}+6|4| 4324 \mid$ I9

Fax +6I 3 959| 9293

Email barry.rawicki@monash.edu
Objective: Hereditary spastic paraplegia (HSP) is a rare progressive disorder with few treatment options. We aim to describe the effect of continuous intrathecal baclofen (ITB) pump therapy on the clinical and functional outcomes of patients with HSP.

Methods: This is a retrospective study, using medical record audit data. Adult patients with HSP who had received ITB trial or therapy and had pre- and post-ITB assessment data available were eligible for inclusion. A purposefully designed audit tool was used. Patients with a successful trial received an ITB implantable SynchroMed ${ }^{\mathbb{B}}$ II pump. Demographic, clinical, and outcome data were obtained pre- and post-pump trial and pump insertion. Functional, spasticity, and mobility measures were compared pre- and post-ITB trial and pre- and post-ITB pump insertion.

Results: Data for nine patients were available. Six were male and the median age was 55 years (Q1, Q3: 46, 55). All received an ITB trial, and those who responded favorably $(\mathrm{n}=8)$ had an ITB pump inserted. Following ITB therapy, improvements were demonstrated for rectus femoris $(P=0.04)$ and gastrocnemius spasticity measures $(P=0.03)$. All patients reported subjective improvements in function, and three of the four with pre- and post-pump assessments, demonstrated clinically meaningful improvements in mobility. Side effects were minimized with appropriate dose titrations.

Conclusion: This is the largest retrospective patient study in the field. The potential benefits of ITB in selected patients with HSP were demonstrated.

Keywords: baclofen, intrathecal baclofen, hereditary spastic paraplegia, gait analysis

\section{Introduction}

Hereditary spastic paraplegia (HSP) refers to a rare group of conditions with a prevalence of 2.7-9.6 per 100,000 population. ${ }^{1,2}$ It may be inherited in an autosomal dominant, autosomal recessive, or X-linked fashion ${ }^{3}$ and manifests as predominantly lower limb, progressive, and bilateral spastic paraparesis. ${ }^{3}$ Although genetic testing is increasingly available it does not yet include all genes known to cause HSP. This means that the absence of a genetic mutation does not exclude the clinical diagnosis of HSP. $^{4}$ Approximately $50 \%$ of people diagnosed with HSP do not have an identifiable genetic cause. Age of onset varies from early childhood to late in life, ${ }^{5}$ as does rate of progression and disease severity. ${ }^{3}$ Consequently, patients with HSP have substantially impaired health-related quality of life (HRQoL). ${ }^{6}$

Treatment is aimed at symptomatic relief as no therapy has been shown to reduce disease progression. Oral antispasticity drugs (eg, baclofen, dantrolene, and diazepam) are common firstline measures. However, there have been no substantial clinical 
trials of oral antispasticity agents in HSP. ${ }^{7}$ Baclofen is a gamma-aminobutyric acid $\mathrm{B}$ receptor agonist that depresses spinal reflexes in order to control skeletal muscle spasticity. ${ }^{8}$ Baclofen crosses the blood-brain barrier poorly, and so high systemic doses are frequently required in order to obtain a clinical effect, ${ }^{8}$ often with unwanted side effects such as drowsiness, weakness, and hypotension. ${ }^{9,10}$ Administration of baclofen via the intrathecal route can reduce side effects and allow for much higher concentrations in the central nervous system where it has its main pharmacological effect. ${ }^{9}$

Intrathecal baclofen (ITB) has been shown to be effective in reducing spasticity in other clinical populations..$^{9,10}$ However, studies specific to HSP patients are limited to small case series. ${ }^{11-13}$ Comparability between studies is also difficult due to a lack of consensus on how best to measure treatment outcomes in this group. Instrumented gait analysis was used by Molteni et $\mathrm{al}^{11}$ in a 2 -year follow-up case study. However, Klebe et $\mathrm{al}^{14}$ found that gait analysis alone did not correlate with clinical response to ITB. This, along with the heterogeneity of the disorder itself, makes it difficult to gain a clear picture of the effectiveness of continuous ITB in HSP. Further research is needed to identify individuals who are likely to benefit from treatment; monitor progress over time; inform dosage schedules; and characterize the side effect profile of this treatment.

In Victoria, Australia, the majority of adult patients with HSP, considered suitable for continuous ITB pump therapy, are placed under the private care of a single clinician considered to be the leading expert in this field, HB Rawicki (HBR). This has provided an opportunity to review a sample of case notes that contain detailed functional, physical, and gait assessment data pre-and post-ITB pump insertion. The aims of our study are to describe the effect of continuous ITB pump therapy on the clinical and functional outcomes of patients with HSP.

\section{Methods}

\section{Patient selection}

The medical records of a single rehabilitation specialist (HBR) were audited. Adult patients ( $>18$ years of age) with a genetic and/or clinical diagnosis of HSP who had received an ITB trial were eligible for inclusion. HSP was diagnosed clinically as a progressive, predominantly lower limb spasticity in the setting of normal neuroimaging of the brain and spine. Testing for very long-chain fatty acids was normal in all participants and basic blood workup for reversible causes was normal. Genetic confirmation was only available in three participants. Those included in our study had a long history of the disease as ITB is considered to be a late treatment option. Patients were excluded if their medical records contained insufficient data. That is, none of the outcome measures were assessable at both baseline and follow-up. Figure 1 provides a consort diagram outlining the screening process. The study protocol was approved by the Human Research and Ethics Committee of Monash University (Approval number: CF13/1365-2013000690) and Epworth HealthCare (Approval number: LR 116-13). Since this study involved secondary use of data that was de-identified at time of collection, informed consent was not required.

\section{Data collection tool}

A literature review was undertaken to identify relevant standardized parameters commonly used in clinical practice to measure treatment outcomes in HSP for which data could be extracted from the medical records. A purpose-designed data collection tool was developed, pilot tested using sample records, and adjusted accordingly. Two records (22\%) were double-audited and compared for reliability. Discrepancies were reviewed and used to further modify the audit tool. A data dictionary and instruction manual were also developed to ensure standardized data collection and to allow for future use of the tool. Data were entered into an Access database (Microsoft Corporation, Redmond, WA, USA). All physical examination and spatial-temporal parameter entries were double-checked by a second researcher, with any discrepancies reviewed and corrected.

\section{Data collection}

Patients' medical records, including clinical gait analysis reports, were audited by a single medical student. The first recorded contact was when patients were referred to the rehabilitation specialist (HBR) for ITB therapy. Demographic data and medical history were extracted from the patient notes. History findings included employment status, musculoskeletal pain, falls history, Walking Index for Spinal Cord Injury (WISCI II) scale, ${ }^{15}$ Functional Ambulation Classification, ${ }^{16}$ Functional Mobility Scale, ${ }^{17}$ and bladder and bowel function.

Gait analyses and physical examinations were performed by physiotherapy staff at various time points. These were generally prior to the ITB trial, post-trial, and then at 6,12 , and 18 months post-pump insertion. In situations where a pre-pump examination was not performed, the pre-trial examination was used as a proxy, provided that this examination was performed within 12 months of pump insertion. The physical examination included goniometric measurements of 


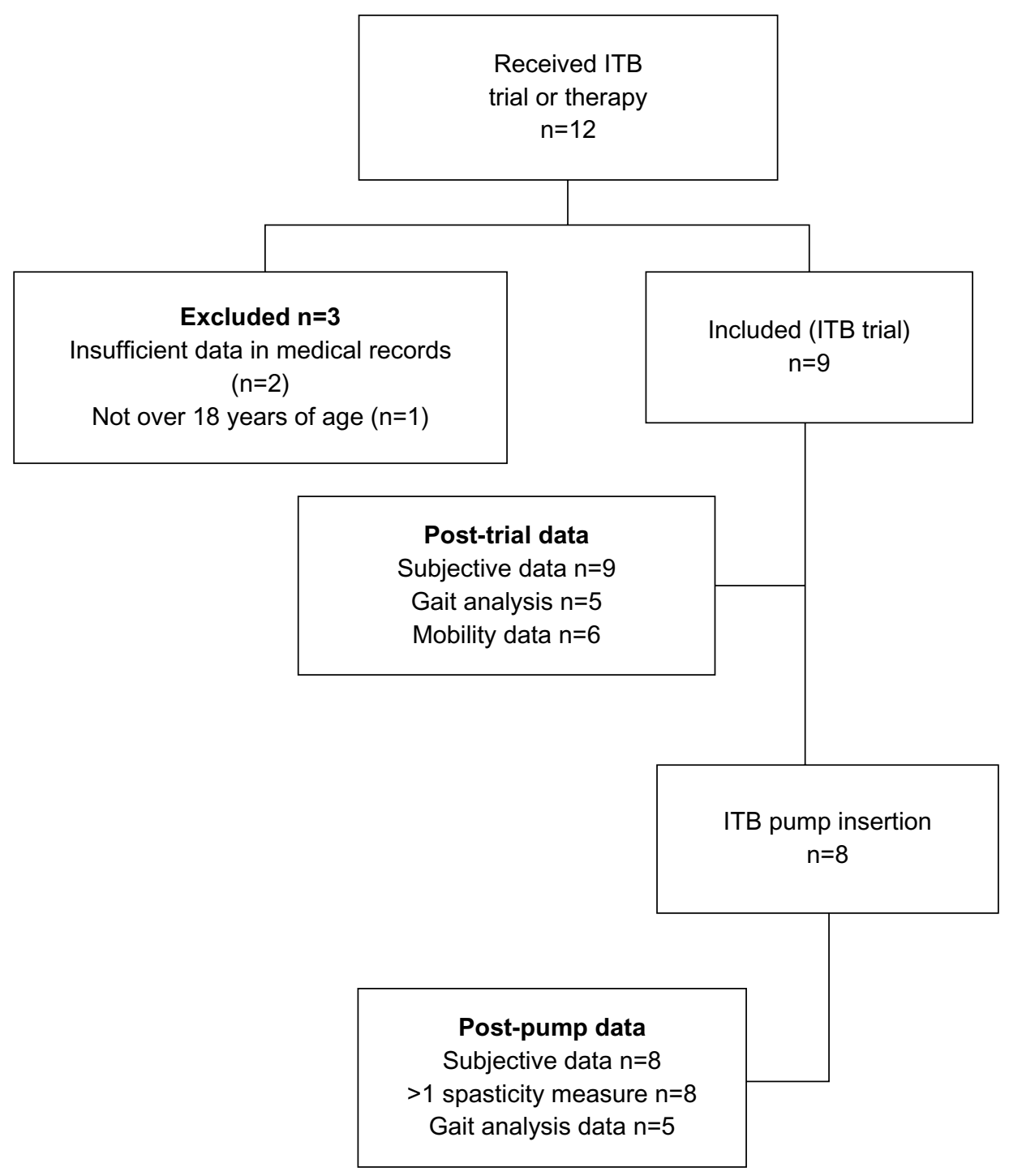

Figure I Flow diagram of the medical record screening process, patient inclusion criteria and patient follow-up. Abbreviation: ITB, intrathecal baclofen.

range of motion, spasticity measured by the Tardieu scale and Modified Ashworth scale, ${ }^{18}$ and muscle strength using the Medical Research Council Grades. ${ }^{19}$ Mobility assessments included the Timed Up and Go Test, the 10 Meter Walk test (10 MWT), and the Step Test. ${ }^{20}$ The 10 MWT was conducted at a self-selected walking speed. A clear pathway of 14 $\mathrm{m}$ was used, with the time and number of steps measured for the intermediated $10 \mathrm{~m}$. All patients were able to walk independently. Assistive devices were used if required and documented from test to test. The velocity was calculated from the time recorded in the 10 MWT (distance divided by time). Spatial-temporal parameters were recorded using instrumental gait analysis.

Patients received ITB trials via a single intrathecal injection. This method was chosen over using a temporary catheter as there is a significant incidence of headache associated with catheter trials which can make testing very difficult and unreliable. The incidence of post-lumbar puncture headache is low, allowing more accurate assessment of spasticity change using this trial method. If the trial was deemed to be successful, a surgically implanted programmable ITB pump and catheter was offered.

\section{Data analysis}

Descriptive statistics were used. The Wilcoxon signed-rank test was used to analyze paired continuous data and the Fisher's exact test was used for categorical data, where sufficient data were available. When multiple pre-therapy outcome measures were available, the most recent value was used in order to minimize the potential effect of disease progression. 
When multiple post-trial outcome measures were available, the earliest value was included in the analysis in order to minimize the potential effect of drug clearance. If a patient underwent multiple trials, the most recent trial was included in the analysis. Data were analyzed using STATA 12.0 for Windows (Stata Corporation, College Station, TX, USA).

\section{Results}

A total of 12 patients were listed as having received either an ITB trial or ITB pump therapy. Of these, nine were eligible for inclusion in the final audit (Figure 1). Most were male and the median age at disease onset was 41 years (range: 16-50 years) and median age at the time of their first ITB trial was 54 years (range: $25-60)$. Three patients (33\%) had a genetically confirmed diagnosis of HSP and one patient had a "complicated" HSP diagnosis (Table 1). Four patients (44\%) were recorded as having slowly progressive disease, while the rate of progression was not recorded for the remaining five patients. All patients had received oral baclofen treatment prior to ITB therapy. Oral baclofen doses ranged from $30 \mathrm{mg}$ (10 $\mathrm{mg}$ three times a day) up to $60 \mathrm{mg}$ (15 mg four times a day) as tolerated. In some patients even the smaller dose was not tolerated due to side effects. A number of patients had received previous non-pharmacological therapies (Table 2) and most reported at least one comorbidity, the most common being musculoskeletal disorders $(n=6)$. At baseline six patients reported some level of urinary urgency and seven patients reported experiencing falls of varying frequency (Table 1).

All nine patients received an ITB trial. Seven patients had one ITB trial only, while one patient had two trials and one

Table I Baseline characteristics of audited patients

\begin{tabular}{ll}
\hline Baseline characteristics & $\mathbf{n ~ ( \% )}$ \\
\hline Male & $6(67)$ \\
Age (years) at disease onset median (QI, Q3) & $4 \mathrm{I}(39,45)$ \\
Age (years) at pump insertion median (QI, Q3) & $55(46,55)$ \\
Employed & $6(67.6)$ \\
Genetic diagnosis recorded & $3(33)$ \\
Complicated HSP recorded & $\mathrm{I}(\mathrm{II})$ \\
History of falls & $7(78)$ \\
Bladder and bowel dysfunction & \\
Urgency recorded only & $\mathrm{I}(\mathrm{II})$ \\
Urgency, nocturia, and incontinence recorded & $\mathrm{I}(\mathrm{II})$ \\
No bladder or bowel symptoms & $3(33)$ \\
Urgency and incontinence recorded & $2(22)$ \\
Urgency, retention, and incontinence recorded & $\mathrm{I}(\mathrm{II})$ \\
Urgency and retention recorded & $\mathrm{I}(\mathrm{II})$ \\
WISCI II score median (QI, Q3) & $20(19,20)$ \\
\hline Abbreiatons:HSP heredary spstc &
\end{tabular}

Abbreviations: HSP, hereditary spastic paraplegia; WISCI, Walking Index for Spinal Cord Injury.
Table 2 Non-pharmacological therapy received by participants prior to intrathecal baclofen therapy

\begin{tabular}{ll}
\hline Therapy & $\mathbf{n}(\%)$ \\
\hline Physiotherapy only & $\mathrm{I}(\mathrm{II})$ \\
Orthoses only & $3(33)$ \\
Physiotherapy and orthoses & $2(22)$ \\
Yoga only & $\mathrm{I}(\mathrm{II})$ \\
No therapy recorded & $2(22)$ \\
Total & $9(100)$ \\
\hline
\end{tabular}

patient had four. Trials were repeated if the previous one had produced unwanted side effects such as paresis, difficulty walking, headache, nausea and vomiting, or loss of balance. Five patients had pre- and post-trial instrumental gait analysis whereas preand post-trial mobility outcome data were available for six patients. One patient demonstrated a marked deterioration in gait measurements following their ITB trial. Two demonstrated a minimal effect and three demonstrated marked improvements in one or more parameters (Table 3). The patient who demonstrated deterioration in gait function did not subsequently have a pump inserted. This patient was older than the other patients (61 years vs median 55 years) had a genetic diagnosis of HSP, a lower WISCI II score (18 vs a median of 20), and was experiencing bladder and bowel symptoms.

Of the eight who went on to receive an implantable ITB pump, all had a SynchroMed ${ }^{\circledR}$ II pump (Medtronic Australasia, Sydney, Australia) inserted. The median disease duration at pump insertion was 11 years $(\mathrm{Q} 1, \mathrm{Q} 3: 8,13)$ and the median follow-up period was 3.4 years (Q1, Q3: 2, 5). All patients received the ITB pump within 2-14 months following a successful trial (median: 6 months) and all reported some degree of subjective improvement in short- and longterm outcomes.

There were no differences between pre- and post-pump measures for the WISCI II scale, Functional Ambulation Classification, Functional Mobility Scale or employment status and no objective effect on bladder or bowel function. However, three patients reported a reduction in the frequency of falls following pump insertion and six reported subjective improvements in mobility.

Post-pump improvements were demonstrated for some, but not all of the physical assessment parameters. The five patients who had the spasticity angle of their left rectus length measurement pre- and post-trial assessment showed a statistically significant improvement (median 43 degrees, Q1, Q3: 35, 65; $P=0.04)$ and seven patients (88\%) showed a statistically significant median reduction of 10 degrees (Q1, Q3: 0, 10; $P=0.03$ ) in the spasticity angle of their right gastrocnemius from pre- to post-trial assessment. 


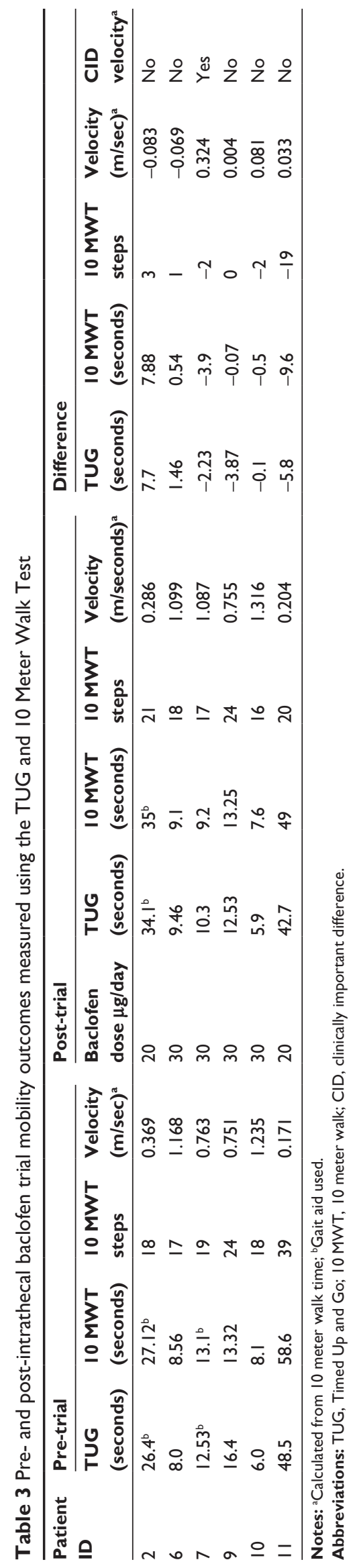

Short-term post-pump mobility outcome data were available for only four of the eight patients, performed a median of 10 days (minimum 6, maximum 13 days) after pump insertion. One patient demonstrated reductions in their mobility parameters. However, the other three showed improvements in all three parameters and demonstrated clinically significant improvements in gait velocity ${ }^{21}$ (Table 4). Longer term post-pump gait analysis was available for five patients. Improvements in gait velocity were still evident in four of the five patients at six months post-pump insertion. Three patients had gait analysis at 12 or 18 months. In two of these, gait velocity had slowed, but was still faster than pre-pump levels (Figure 2).

The median ITB trial dose was $30 \mu \mathrm{g} /$ day (range: $20-40$ ). This dose was chosen because, based on the clinical experience of author HBR, people with HSP often develop weakness with higher doses (medium $50 \mu \mathrm{g}$ ) even at the trial phase. He has found the lower dose of $30 \mu \mathrm{g}$ to be adequate and appropriate for trials. The median pump dose at commencement of therapy was $46.5 \mu \mathrm{g} /$ day (Q1, Q3: 38, 54). Pump doses were progressively increased or decreased in accordance with symptom control. After 1 year of having a pump, the maximum pump dose for a patient was $705 \mu \mathrm{g} /$ day and the minimum pump dose was $65 \mu \mathrm{g} /$ day. Side effects were experienced to some degree by all patients following pump insertion but were able to be resolved by dose titration. Side effects included headache, meningism, nausea and vomiting, baclofen tolerance, constipation, lower limb numbness, fatigue, haziness, diplopia, paresis, difficulty mobilizing, and loss of comfort when using orthosis. Two patients experienced significant side effects, one at a dosage of $45 \mu \mathrm{g} /$ day and the other at $130 \mu \mathrm{g} /$ day, thereby requiring their dose to be reduced to zero. Once the side effects had subsided, their dosage was slowly and successfully recommenced.

\section{Discussion}

This is the largest retrospective study in which the clinical and functional outcomes associated with the use of continuous ITB in adults with HSP are described. We provide new and preliminary evidence for the use of continuous ITB in this rare group of patients. Our results showed that appropriately selected patients with HSP, based on responses to an ITB trial, who received an ITB pump, had subjective and clinical improvements in symptoms and gait function. Improvements were sustained for up to 18 months in most patients.

These results are consistent with previous observational studies in patients with HSP and other clinical groups for reducing spasticity ${ }^{12,13}$ and improving gait. ${ }^{11,13,14}$ The majority 


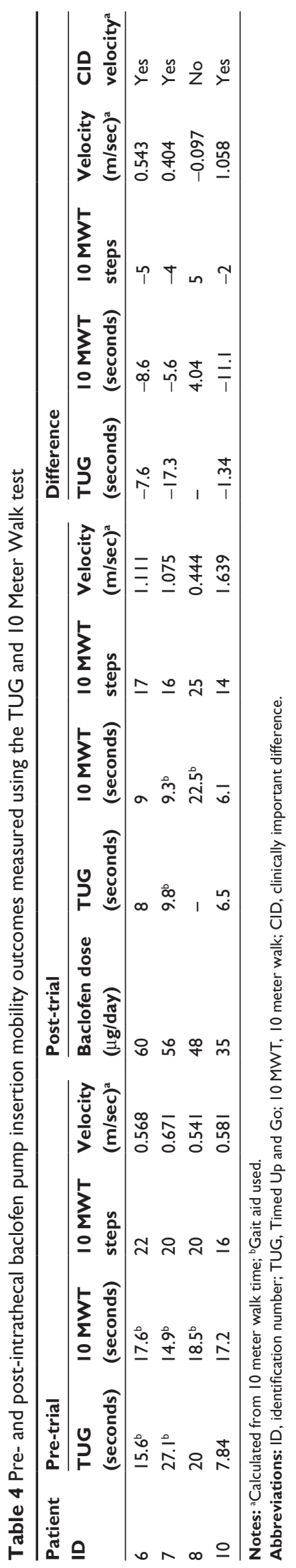

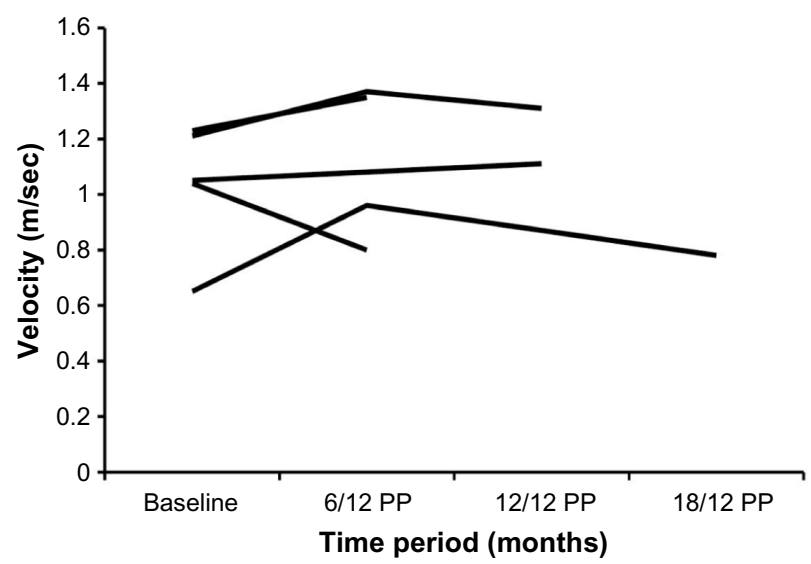

Figure 2 Long-term change in gait velocity following intrathecal baclofen pump insertion, measured using instrumental gait analysis $(n=5)$ participants. Abbreviation: PP, post-pump.

of patients in our study showed clinically significant improvements in functional gait parameters following pump insertion. One proposed mechanism is that ITB may increase the threshold of polysynaptic reflexes allowing a more normal supraspinal motor pattern to emerge. ${ }^{8}$ This is further supported by our data which showed significant reductions in spasticity in the rectus femoris and gastrocnemius muscles for patients who reported improvements in mobility.

Objective measurements of gait, range, and spasticity are important for quantifying the magnitude of the treatment effect. However, it is the subjective improvements and the degree to which these observed changes are of a clinical significance that are most relevant to the patient. Subjective improvements were reported by all patients who went on to have a pump inserted and clinically meaningful differences in gait velocity were demonstrated for three of the four patients who had pre- and post-pump gait data. It is likely that these changes would have resulted, not only in general functional improvements related to independence and activities of daily living, but would impact on patients overall HRQoL. Correlations have been previously demonstrated between walking ability and both mental and physical HRQoL in patients with HSP and disease progression to the point of needing a walking aid was reported as having the greatest negative impact on HRQoL. ${ }^{6}$

Although all of the patients in our study had a clinical diagnosis of HSP, only three had a genetically confirmed diagnosis. Results for these three varied and having a clinical diagnosis only, did not appear to be an indicator for success or failure of ITB therapy. However, as our study contains only a small number of patients diagnosed genetically, it is not possible to determine if those with a genetic diagnosis of HSP differ in their response to ITB. 
The strengths of this study are the comparatively large number of cases audited and the use of a standardized data collection tool that can be used continuously to provide pooled results in the future. Our results build on a growing body of evidence that suggests that continuous ITB pump therapy may be an effective treatment choice for those with HSP for improving gait and maintaining functional independence.

\section{Study limitations}

The main limitations associated with our study are those related to study design and data availability. Since this was a retrospective audit of medical records there is the potential for reporting bias because information recorded by clinicians is not standardized. Consequently, there were a number of missing data items and inconsistencies in the reporting of data at the various time points. Although all patients provided subjective follow-up data, only five had detailed gait analysis data available. Another limitation is that we did not collect the individual dose range of oral baclofen used prior to participation in the trial of ITB in our audit. However, we were able to provide the overall dose ranges. Finally, because HSP is a rare disorder, data were available for only a small number of patients, limiting the ability to perform more complex data analyses and comparisons.

\section{Conclusion}

The use of continuous ITB pump therapy may be effective in improving gait and mobility in selected adults with HSP. This is important given the progressive nature of the disease and the lack of other treatment options for affected patients. Additional evidence from prospective cohort studies with standardized data collection is needed to confirm these results.

\section{Acknowledgments}

Medtronic Inc. provided a grant $(<$ US $\$ 20,000)$ to support development of a database and research assistant time for this project; they had no involvement in the collection, analysis, or interpretation of the data or in the decision to submit the article for publication. Dominique Cadilhac is supported by a National Health and Medical Research Council (NHMRC)/ National Heart Foundation Research Fellowship (1063761) and Nadine Andrew is supported by an NHMRC Early Career Fellowship (1072053). Jessie Khera was supported by a medical academic scholarship from Monash Health.

\section{Disclosure}

The authors report no conflicts of interest in this work.

\section{References}

1. Braschinsky M, Lüüs S-M, Gross-Paju K, Haldre S. The prevalence of hereditary spastic paraplegia and the occurrence of SPG4 mutations in Estonia. Neuroepidemiology. 2009;32(2):89-93.

2. Coutinho P, Ruano L, Loureiro J, et al. Hereditary ataxia and spastic paraplegia in Portugal. JAMA Neurol. 2013;70(6):746-755.

3. Contino G, Novelli G. Hereditary spastic paraplegia: clinical genomics and pharmacogenetic perspectives. Expert Opin Pharmacother. 2006;7(14):1849-1856.

4. Fink JK. Hereditary spastic paraplegia overview. In: Pagon RA, Adam MP, Ardinger HH, et al, editors. GeneReviews(R). Seattle (WA): University of Washington; 1993.

5. Werselin 1, Keiding N. Hereditary ataxias: epidemiological aspects. Neuroepidemiology. 1990;9(6):321-331.

6. Klimpe S, Schüle R, Kassubek J, et al. Disease severity affects quality of life of hereditary spastic paraplegia patients. Eur J Neurol. 2012;19(1):168-171.

7. Scheuer K, Svenstrup K, Jennum P, et al. Double-blind crossover trial of gabapentin in SPG4-linked hereditary spastic paraplegia. Eur J Neurol. 2007;14(6):663-666.

8. Dan B, Cheron G. Intrathecal baclofen normalizes motor strategy for squatting in familial spastic paraplegia: a case study. Neurophysiol Clin. 2000;30(1):43-48.

9. Meythaler J, Guin-Renfroe S, Brunner R, Hadley M. Intrathecal baclofen for spastic hypertonia from stroke. Stroke. 2001;32(9):2099-2109.

10. Penn R, Savoy $S$, Corcos D, et al. Intrathecal baclofen for severe spinal spasticity. N Engl J Med. 1989;320(23):1517-1521.

11. Molteni F, Carda S, Cazzaniga M, Magoni L, Rossini M, Caimmi M. Instrumental evaluation of gait modifications before and during intrathecal baclofen therapy: a 2-year follow-up case study. Am J Phys Med Rehabil. 2005;84(4):303-306.

12. Bushman W, Steers WD, Meythaler JM. Voiding dysfunction in patients with spastic paraplegia: urodynamic evaluation and response to continuous intrathecal baclofen. Neurourol Urodyn. 1993;12(2):163-170.

13. Meythaler JM, Steers WD, Tuel SM, Cross LL, Sesco DC, Haworth CS. Intrathecal baclofen in hereditary spastic paraparesis. Arch Phys Med Rehabil. 1992;73(9):794-797.

14. Klebe S, Stolze H, Kopper F, et al. Objective assessment of gait after intrathecal baclofen in hereditary spastic paraplegia. J Neurol. 2005;252(8):991-993.

15. Dittuno P, Dittuno J. Walking index for spinal cord injury (WISCI II): scale revision. Spinal Cord. 2001;39(12):654-656.

16. Kollen B, Kwakkel G, Lindeman E. Time dependency of walking classification in stroke. Phys Ther. 2006;86(5):618-625.

17. Graham H, Harvey A, Rodda J, Nattrass G, Pirpiris M. The Functional Mobility Scale (FMS). J Pediatr Orthop. 2004;24(5):514-520.

18. Bohannon R, Smith M. Interrater reliability of a modified Ashworth scale of muscle spasticity. Phys Ther. 1987;67(2):206-207.

19. Medical Research Council. Aids to the Investigation of the Peripheral Nervous System. In: Her Majesty's Stationary Office, ed. London: Medical Research Council; 1943.

20. Lam T, Noonan V, Eng J; SCIRE Research Team. A systematic review of functional ambulation outcome measures in spinal cord injury. Spinal Cord. 2008;46(4):246-254.

21. Tilson J, Sullivan K, Cen S, et al. Meaningful gait speed improvement during the first 60 days poststroke: minimal clinically important difference. Phys Ther. 2010;90(2):196-208. 
Clinical Audit

Dovepress

\section{Publish your work in this journal}

Clinical Audit is an international, peer-reviewed, open access journal focusing on the processes and outcomes of clinical audit in any area of healthcare. All aspects of patient care are addressed within the journal and practitioners from all disciplines are invited to submit their work. Areas covered include: Publication of audits; How an audit has changed practice; changed patient care; Calls and justifications for new audits. The manuscript management system is completely online and includes a very quick and fair peer-review system, which is all easy to use. Visit http://www.dovepress. com/testimonials.php to read real quotes from published authors.

Submit your manuscript here: http://www.dovepress.com/clinical-audit-journa 